\title{
Metformin use is associated with a lower risk of osteoporosis/vertebral fracture in Taiwanese patients with type 2 diabetes mellitus
}

\author{
Chin-Hsiao Tseng(10) 1,2,3 \\ ${ }^{1}$ Department of Internal Medicine, National Taiwan University College of Medicine, Taipei, Taiwan, ${ }^{2}$ Division of \\ Endocrinology and Metabolism, Department of Internal Medicine, National Taiwan University Hospital, Taipei, \\ Taiwan, and ${ }^{3}$ Division of Environmental Health and Occupational Medicine of the National Health Research \\ Institutes, Zhunan, Taiwan
}

Correspondence should be addressed to C-H Tseng Email

ccktsh@ms6.hinet.net

\begin{abstract}
Objectives: To investigate the metformin effect on the risk of osteoporosis (OS) and/or vertebral fracture (VF). Methods: We enrolled 14611 pairs of metformin ever and never users matched on propensity score (PS) from Taiwan's National Health Insurance database. All patients had new-onset type 2 diabetes mellitus (T2DM) during 1999-2005 and were free from OS and/or any fracture at the start of follow-up on January 1, 2006. They were followed up until December 31, 2011 for the incidence of OS/VF. Cox regression incorporated with the inverse probability of treatment weighting using PS was used in the main analyses.

Results: New-onset OS/VF was diagnosed in 1757 never users (median follow-up 5.0 years) and 1143 ever users (median follow-up 5.3 years). The respective incidence rates were 2870.97 and 1713.20 per 100000 person-years. Two-thirds of the incident cases had OS without VF and the other third had VF. In main analyses, the hazard ratio for ever vs never users was 0.592 (95\% Cl: 0.550-0.638). In either sex, a dose-response pattern was noted and metformin therapy $>2$ years was consistently associated with a lower risk. The protective effect attenuated with increasing age but remained significant in patients aged $\geq 80$ years. In sensitivity analyses, metformin significantly reduced the risk of both OS and VF (with or without a prior OS) by 30-40\%. Additional analyses showed a null association for other antidiabetic drugs, but significant interactions between metformin and insulin, sulfonylurea and pioglitazone, respectively, were noted.
\end{abstract}

Conclusion: Metformin use is associated with a lower risk of OS/VF.

\section{Introduction}

Patients with diabetes mellitus, either type 1 (T1DM) or type 2 (T2DM), suffer from a significantly higher risk of osteoporosis, putting them at a high risk of vertebral fracture $(1,2,3,4,5)$. However, the pathophysiology of bone changes associated with T1DM and T2DM may not be the same $(1,6)$. Insulin exerts osteogenic effects (7) and patients with T1DM, which mainly manifests in (c) 2021 European Society of Endocrinology Printed in Great Britain adolescence, may have impaired bone formation because of inadequate accrual of peak bone mass resulting from insulin deficiency (1). On the other hand, T2DM mainly affects adult populations and patients with T2DM may have a higher bone mineral density than nondiabetic people because of the bone anabolic effect of hyperinsulinemia resulting from insulin resistance (1, 
2). Despite the normal or higher levels of bone mineral density, patients with T2DM have a higher risk of fracture because of poor bone quality and increased adipogenicity in bone tissue (1).

Patients who use antidiabetic drugs such as insulin $(8,9,10)$, thiazolidinediones $(9,10,11,12)$ and canagliflozin (13) may have a higher risk of fracture. The higher risk in insulin users may be explained by the increased incidence of hypoglycemia-induced falls (10). Thiazolidinediones increase fracture risk because it may shift the differentiation of mesenchymal stem cells to adipocytes via the activation of peroxisome proliferatoractivated receptor gamma $(1,2)$. Diabetes patients with chronic microvascular complications may suffer from a higher risk of fracture because chronic renal insufficiency can affect bone metabolism (14) and retinopathy or neuropathy may increase the risk of fall because of visual impairment and/or frailty $(1,2,3,4,5,15,16)$.

Some studies from the USA $(8)$ and Denmark $(17,18)$ suggested that metformin users might have a lower risk of fracture $(8,17)$. However, a lack of association was observed in a case-control study in Italy (19), a cross-sectional study in Japan (9) and the Osteoporotic Fractures in Men study in the USA (20). Two meta-analyses were published in $2019(10,21)$. The first estimated relative risk of 0.86 (95\% CI: 0.75-0.99) for any fracture for metformin users, but no significant risk reduction of vertebral fracture was observed (10). The other meta-analysis estimated a pooled relative risk of 0.82 (95\% CI: $0.72-0.93)$ for any fracture associated with metformin use, but the site-specific fracture was not evaluated (21). Therefore, whether metformin use may reduce the risk of vertebral fracture has not been extensively investigated and the findings are not conclusive. Furthermore, no previous studies have ever investigated the effect of metformin on the risk of osteoporosis.

By using a nationwide reimbursement database of Taiwan's National Health Insurance (NHI), the present study compared the risk of osteoporosis and/or vertebral fracture in ever users vs never users of metformin in a well-matched cohort based on propensity score (PS) in patients with T2DM.

\section{Subjects and methods}

\section{The national health insurance}

The NHI is a universal and compulsory healthcare system implemented in Taiwan since March 1995. It covers $>99 \%$ of the population and the Bureau of the NHI has contracts with all hospitals and 93\% of all medical settings. The NHI reimbursement database was described in more detail in a previously published paper (22). In brief, it keeps all disease diagnoses, medication prescriptions and clinical procedures as computer records that can be used for academic research after ethics review and approval. The present study was granted approval number 99274 by the National Health Research Institutes.

\section{Outcome definitions}

During the study period, disease diagnoses were coded by the International Classification of Diseases, Ninth Revision, Clinical Modification (ICD-9-CM). The ICD9-CM codes for the disease diagnoses used in the present study are provided in Supplementary Table 1 (see section on supplementary materials given at the end of this article).

We considered osteoporosis and/or vertebral fracture diagnosed at outpatient clinics or during hospitalization as a composite outcome investigated in the main analyses. The ICD-9-CM codes for osteoporosis included 733.00 (osteoporosis unspecified), 733.01 (senile osteoporosis), 733.02 (idiopathic osteoporosis), 733.03 (disuse osteoporosis) and 733.09 (other osteoporosis). Vertebral fracture was based on the ICD-9-CM codes of 805 (fracture of vertebral column without mention of spinal cord injury) and 806 (fracture of vertebral column with spinal cord injury).

In sensitivity analyses, osteoporosis/vertebral fracture as a composite outcome or osteoporosis and vertebral fracture separately (either diagnosed at outpatient clinics and/or during hospitalization or during hospitalization only) were considered as the outcome. Because a primary diagnosis made during hospitalization should have been supported by laboratory examinations for reimbursement purpose, it was considered more accurate than a diagnosis made at outpatient clinics.

\section{Study population}

A PS-matched cohort of ever and never users of metformin were enrolled according to the procedures shown in Fig. 1. At first, 423949 newly diagnosed diabetes patients during 1999-2005 who had been followed up in the outpatient clinics with prescriptions of antidiabetic drugs for two or more times were identified from the whole nationwide database. Ineligible patients were then excluded: (1) metformin ever users who had been treated with other antidiabetic drugs before metformin was first prescribed 


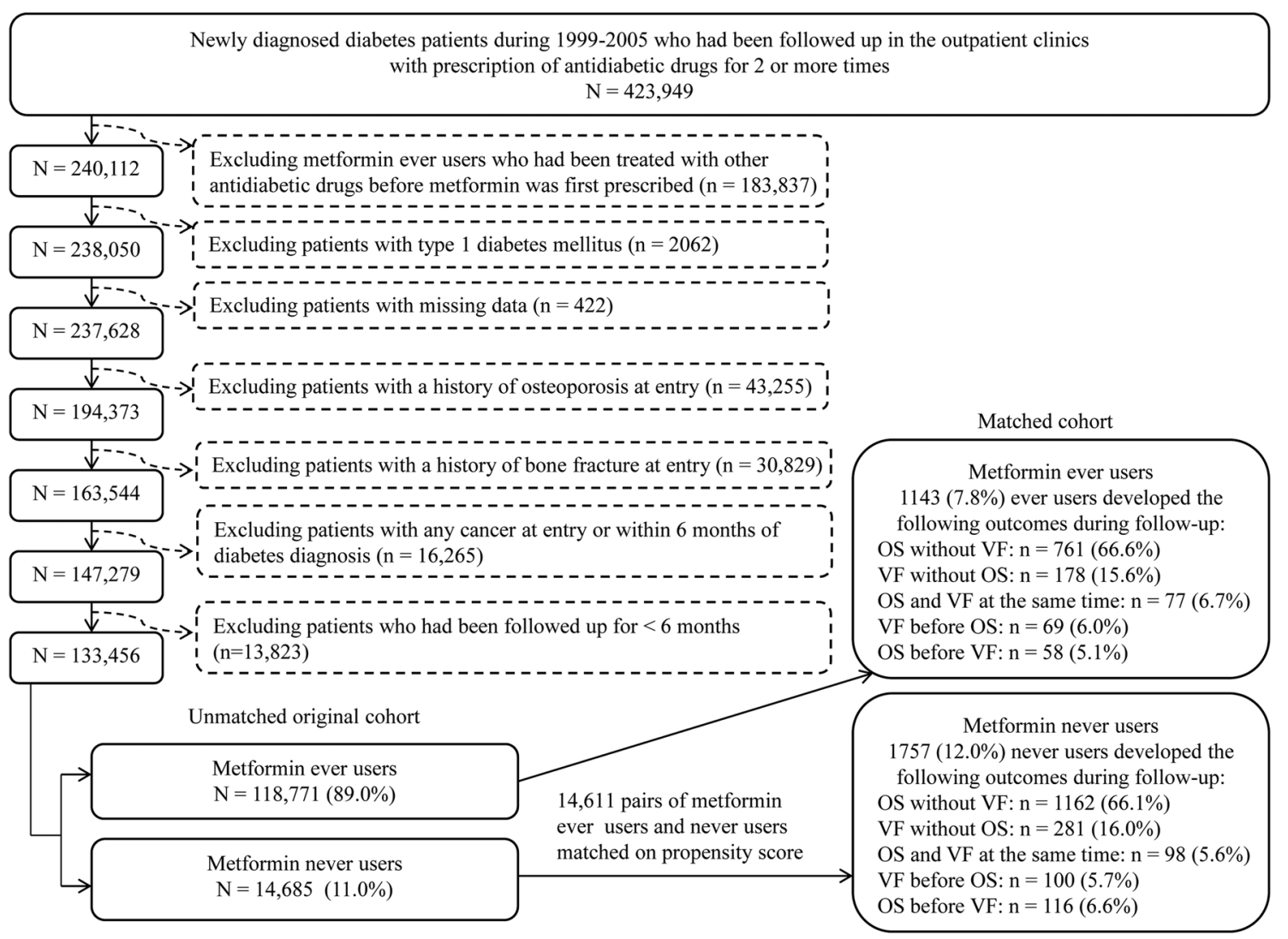

\section{Figure 1}

Flowchart showing the procedures in creating a cohort of 1:1 propensity score-matched pairs of ever and never users of metformin from the reimbursement database of the National Health Insurance. Outcomes investigated in the main analyses include osteoporosis (OS) and/or vertebral fracture (VF) diagnosed at outpatient clinics or during hospitalization.

( $n=183$ 837, to avoid the carry-over effects of other antidiabetic drugs); (2) patients with T1DM ( $n=2062$, these patients may have pathophysiological bone changes different from patients with T2DM and metformin is not indicated for use in patients with T1DM); (3) patients with missing data $(n=422)$; (4) patients with a history of osteoporosis at entry $(n=43255$, these patients were excluded because osteoporosis was one of the outcomes investigated), (5) patients with a history of any bone fracture at entry ( $n=30829$, these patients were excluded because vertebral fracture was one of the outcomes investigated); (6) patients with any cancer at entry or within 6 months of diabetes diagnosis $(n=16265$, these patients were excluded because cancer may affect bone fracture and the estimation of follow-up person-years could be biased because of shortened lifespan); and (7) patients who had been followed up for $<6$ months ( $n=13823$, to exclude the immortal time bias resulting from the short follow-up period of the initial 6 months). As a result, 118771 ever users and 14685 never users of metformin were identified as an unmatched original cohort. Logistic regression was used to create PS from all characteristics listed in Table 1 plus the date of entry. A matched cohort of 14611 pairs of ever users and never users was then created from the unmatched original cohort by matching the PS using the Greedy 8 --> 1 digit match algorithm (23).

\section{Data collection}

The following categories of variables were considered as factors that might potentially lead to confounding by indication and were included in the estimation of PS used in the above matching procedures and in the estimation of hazard ratios by Cox regression incorporated with the inverse probability of treatment weighting (IPTW) using the PS (described in the Supplementary document). They were also treated as potential confounders in traditional multiple Cox regression and in multiple logistic regression. The ICD-9-CM codes of the related disease diagnoses can be seen in Supplementary Table 1. The accuracy of 
Table 1 Characteristics in never and ever users of metformin. Data are present as $n(\%)$.

\begin{tabular}{|c|c|c|c|}
\hline Variables & Never users $(n=14,611)$ & Ever users $(n=14,611)$ & S.D. \\
\hline \multicolumn{4}{|l|}{ Basic information } \\
\hline Age* (years) & $62.17 \pm 13.32$ & $62.01 \pm 12.29$ & -0.66 \\
\hline Years since diabetes diagnosis* & $4.10 \pm 2.48$ & $4.10 \pm 2.10$ & -0.16 \\
\hline Sex (men) & $9073(62.10)$ & $9062(62.02)$ & -0.18 \\
\hline \multicolumn{4}{|l|}{ Occupation } \\
\hline I & $5571(38.13)$ & $5688(38.93)$ & \\
\hline II & $2597(17.77)$ & $2607(17.84)$ & 0.08 \\
\hline III & $3210(21.97)$ & $3240(22.18)$ & 0.57 \\
\hline IV & $3233(22.13)$ & $3076(21.05)$ & -2.58 \\
\hline \multicolumn{4}{|l|}{ Living region } \\
\hline Taipei & $5187(35.50)$ & $5171(35.39)$ & \\
\hline Northern & $1550(10.61)$ & $1545(10.57)$ & -0.10 \\
\hline Central & $2468(16.89)$ & $2467(16.88)$ & 0.00 \\
\hline Southern & $2466(16.88)$ & $2561(17.53)$ & 1.79 \\
\hline Kao-Ping and Eastern & $2940(20.12)$ & $2867(19.62)$ & -1.25 \\
\hline \multicolumn{4}{|l|}{ Major comorbidities } \\
\hline Hypertension & $10,966(75.05)$ & $10,909(74.66)$ & -0.79 \\
\hline Dyslipidemia & $8536(58.42)$ & $8553(58.54)$ & 0.31 \\
\hline Obesity & $264(1.81)$ & $268(1.83)$ & 0.10 \\
\hline \multicolumn{4}{|l|}{ Diabetes-related complications } \\
\hline Nephropathy & $3587(24.55)$ & $3459(23.67)$ & -2.30 \\
\hline Eye diseases & $1355(9.27)$ & $1372(9.39)$ & -0.03 \\
\hline Diabetic polyneuropathy & $3920(26.83)$ & $3845(26.32)$ & -1.11 \\
\hline Stroke & $1471(10.07)$ & 1466 (10.03) & -0.44 \\
\hline Ischemic heart disease & $5757(39.40)$ & $5700(39.01)$ & -0.80 \\
\hline Peripheral arterial disease & $2218(15.18)$ & $2193(15.01)$ & -0.59 \\
\hline \multicolumn{4}{|l|}{ Antidiabetic drugs } \\
\hline Insulin & $1171(8.01)$ & $1144(7.83)$ & -2.64 \\
\hline Sulfonylurea & $10728(73.42)$ & $11039(75.55)$ & 4.00 \\
\hline Meglitinide & $1222(8.36)$ & $1231(8.43)$ & -0.17 \\
\hline Acarbose & $1563(10.70)$ & $1640(11.22)$ & -0.17 \\
\hline Rosiglitazone & $443(3.03)$ & $432(2.96)$ & -1.38 \\
\hline Pioglitazone & $346(2.37)$ & $350(2.40)$ & -0.69 \\
\hline \multicolumn{4}{|l|}{ Common comorbidities } \\
\hline COPD & $5872(40.19)$ & $5906(40.42)$ & 0.62 \\
\hline Tobacco abuse & $194(1.33)$ & 185 (1.27) & -0.56 \\
\hline Alcohol-related diagnoses & $713(4.88)$ & $661(4.52)$ & -1.75 \\
\hline \multicolumn{4}{|l|}{ Medications used by diabetes patients } \\
\hline ACE inhibitor/angiotensin receptor blocker & $8907(60.96)$ & $8828(60.42)$ & -1.21 \\
\hline Calcium channel blocker & $8322(56.96)$ & $8208(56.18)$ & -1.42 \\
\hline Statin & $5602(38.34)$ & $5525(37.81)$ & -1.28 \\
\hline Fibrate & $3816(26.12)$ & $3738(25.58)$ & -1.32 \\
\hline Aspirin & $7100(48.59)$ & $7075(48.42)$ & -0.26 \\
\hline \multicolumn{4}{|l|}{ Other potential confounders } \\
\hline Heart failure & $2409(16.49)$ & 2409 (16.49) & -0.84 \\
\hline Parkinson's disease & $401(2.74)$ & $378(2.59)$ & -0.86 \\
\hline Dementia & $843(5.77)$ & $819(5.61)$ & -0.67 \\
\hline Head injury & $123(0.84)$ & $107(0.73)$ & -1.33 \\
\hline Valvular heart disease & $1301(8.90)$ & $1281(8.77)$ & -0.36 \\
\hline Hypoglycemia & $240(1.64)$ & $216(1.48)$ & -1.36 \\
\hline H. pylori infection & $3041(20.81)$ & $2903(19.87)$ & -2.36 \\
\hline Epstein-Barr virus infection & $66(0.45)$ & $78(0.53)$ & 1.13 \\
\hline Hepatitis B virus infection & $245(1.68)$ & $247(1.69)$ & 0.06 \\
\hline Hepatitis C virus infection & $508(3.48)$ & $480(3.29)$ & -1.09 \\
\hline HIV disease & $9(0.06)$ & $5(0.03)$ & -1.55 \\
\hline Cirrhosis of liver without mention of alcohol & $672(4.60)$ & $680(4.65)$ & 0.12 \\
\hline Other chronic nonalcoholic liver disease & $1024(7.01)$ & $1048(7.17)$ & 0.53 \\
\hline
\end{tabular}


Table 1 Continued.

\begin{tabular}{lcc}
\hline Variables & Never users $(n=14,611)$ \\
\hline Autoimmune diseases & $948(6.49)$ \\
Organ transplantation & $79(0.54)$ \\
Insomnia & $2944(20.15)$ \\
Malaise and fatigue & $500(3.42)$ \\
History of some disorders of the CNS & $2752(18.84)$ \\
Immunosuppressants & $655(4.48)$ \\
Hyperthyroidism & $487(3.33)$ \\
\hline
\end{tabular}

\begin{tabular}{c}
\hline Ever users $(n=14,611)$ \\
\hline $868(5.94)$ \\
$62(0.42)$ \\
$2954(20.22)$ \\
$522(3.57)$ \\
$2711(18.55)$ \\
$623(4.26)$ \\
$494(3.38)$ \\
\hline
\end{tabular}

$\begin{array}{r}\hline \text { s.d. } \\ \hline-2.28 \\ -1.93 \\ 0.33 \\ 0.98 \\ -0.60 \\ -1.17 \\ 0.23 \\ \hline\end{array}$

Refer to 'Subjects and methods' for the classification of occupation.

*Data presented as mean \pm S.D.

$A C E$, angiotensin converting enzyme; COPD, chronic obstructive pulmonary disease.

disease diagnoses in the NHI database has been studied previously. Agreements between claim data and medical records are moderate to substantial, with Kappa values ranged from 0.55 to 0.86 (24).

I. Basic information: age, time-elapsed since diabetes diagnosis, sex, occupation and living region. The classifications of living region and occupation were detailed elsewhere (25). In brief, the living region was classified as Taipei, Northern, Central, Southern, and Kao-Ping/Eastern. Occupation was classified as class 1 (civil servants, teachers, employees of governmental or private businesses, professionals and technicians), class 2 (people without a specific employer, self-employed people or seamen), class 3 (farmers or fishermen) and class 4 (low-income families supported by social welfare, or veterans).

II. Major comorbidities: hypertension, dyslipidemia and obesity.

III. Diabetes-related complications: nephropathy, eye diseases, diabetic polyneuropathy, stroke, ischemic heart disease and peripheral arterial disease.

IV. Antidiabetic drugs: insulin, sulfonylurea, meglitinide, acarbose, rosiglitazone and pioglitazone.

V. Commonly encountered comorbidities: chronic obstructive pulmonary disease (a surrogate for smoking), tobacco abuse and alcohol-related diagnoses.

VI. Commonly used medications in diabetes patients: angiotensin-converting enzyme inhibitor/ angiotensin receptor blocker, calcium channel blocker, statin, fibrate and aspirin.

VII. Other potential confounders: heart failure, Parkinson's disease, dementia, head injury, valvular heart disease, hypoglycemia, history of Helicobacter pylori (HP) infection (defined below), Epstein-Barr virus infection, hepatitis B virus infection, hepatitis $C$ virus infection, human immunodeficiency virus disease, cirrhosis of liver without mention of alcohol, other chronic nonalcoholic liver disease, autoimmune diseases, organ transplantation, insomnia, malaise and fatigue, history of some disorders of the CNS, immunosuppressants (consistent use $\geq 90$ days and included corticosteroids, calcineurin inhibitors and/or inosine-5'-monophosphate dehydrogenase inhibitors) and hyperthyroidism. History of HP infection was defined in patients who met one of the following two criteria: (1) patients receiving an HP eradication therapy (detailed previously (26), defined in brief as a combination use of proton pump inhibitor or $\mathrm{H} 2$ receptor blockers, plus clarithromycin, metronidazole or levofloxacin, plus amoxicillin or tetracycline, with or without bismuth, in the same prescription order for 7-14 days); and/or (2) ICD-9-CM code of HP infection.

VIII. In sex-specific analyses that included women only, two additional covariates were included in the estimation of PS or as covariates for adjustment in regression analyses, that is, 'symptomatic menopausal or female climacteric states' and 'use of estrogen'.

\section{Statistical analysis}

Statistical analysis are described in detail in the Supplementary document. SAS statistical software, version 9.3 (SAS Institute, Cary, NC) was used. $P<0.05$ was considered statistically significant. In brief, standardized difference was calculated for each covariate (27), incidence density was calculated and cumulative duration of metformin therapy was used as an indicator of dose-response relationship. Cox regression incorporated with the inverse probability of treatment weighting (28) was used to estimate HRs 
and their 95\% CIs in main analyses and in sensitivity analyses. Traditional Cox regression was used to investigate the interactions between metformin and other antidiabetic drugs. To examine the consistency of the findings, multiple logistic regression was used to analyze the database cross-sectionally. To reduce the risk of overadjustment or unnecessary adjustment (29), additional logistic regression models were created by restricting adjusted variables to those with significant $P$-values between metformin users and non-users and between patients with and without the outcome.

\section{Results}

As shown in Fig. 1, 1143 (7.8\%) of the 14611 ever users of metformin and 1757 (12.0\%) of the 14611 never users of metformin in the matched cohort developed osteoporosis and/or vertebral fracture. Osteoporosis without vertebral fracture was the most common diagnosis, representing approximately two-thirds of the incident cases in either the ever users (761 cases or 66.6\%) or the never users of metformin (1162 cases or $66.1 \%)$. Vertebral fracture could happen either without a diagnosis of osteoporosis (178 cases or $15.6 \%$ in ever users and 281 cases or $16.0 \%$ in never users), at the same time of a diagnosis of osteoporosis (77 cases or $6.7 \%$ in ever users and 98 cases or $5.6 \%$ in never users) or before the diagnosis of osteoporosis (69 cases or $6.0 \%$ in ever users and 100 cases or $5.7 \%$ in never users). Only a small proportion of the incident cases (58 cases or $5.1 \%$ in ever users and 116 cases or $6.6 \%$ in never users) would have a prior diagnosis of osteoporosis before vertebral fracture occurred.

The characteristics in never users and ever users of metformin are shown in Table 1 . The two groups were well matched because all covariates had values of standardized difference $<10 \%$.

Table 2 shows the incidence of osteoporosis/vertebral fracture and the HRs by metformin exposure in both sexes together and in men and women separately in the main analyses. For a median follow-up of 5.0 years in never users and 5.3 years in ever users, 1757 and 1143 cases developed an outcome of osteoporosis/vertebral fracture, and the respective incidence rates were 2870.97

Table 2 Incidence of osteoporosis/vertebral fracture diagnosed at outpatient clinics or during hospitalization and hazard ratios by metformin exposure in main analyses.

\begin{tabular}{|c|c|c|c|c|c|c|c|}
\hline Metformin use & $\begin{array}{l}\text { Incident } \\
\text { case } \\
\text { number }\end{array}$ & $\begin{array}{c}\text { Cases } \\
\text { followed }\end{array}$ & Person-years & $\begin{array}{c}\text { Incidence rate } \\
\text { (per } 100000 \\
\text { person-years) }\end{array}$ & $\begin{array}{c}\text { Hazard } \\
\text { ratio }\end{array}$ & $95 \% \mathrm{Cl}$ & $P$ value \\
\hline \multicolumn{8}{|l|}{ Both sexes } \\
\hline Never users & 1757 & 14611 & 61198.80 & 2870.97 & 1.000 & & \\
\hline Ever users & 1143 & 14611 & 66717.12 & 1713.20 & 0.592 & $0.550-0.638$ & $<0.0001$ \\
\hline \multicolumn{8}{|c|}{ Tertiles of cumulative duration of metformin therapy (months) } \\
\hline Never users & 1757 & 14611 & 61198.80 & 2870.97 & 1.000 & & \\
\hline$<25.5$ & 548 & 4813 & 16403.15 & 3340.82 & 1.145 & $1.039-1.260$ & 0.0060 \\
\hline $25.5-54.8$ & 384 & 4829 & 22559.85 & 1702.14 & 0.584 & $0.523-0.652$ & $<0.0001$ \\
\hline$>54.8$ & 211 & 4969 & 27754.12 & 760.25 & 0.263 & $0.228-0.304$ & $<0.0001$ \\
\hline \multicolumn{8}{|l|}{ Men } \\
\hline Never users & 680 & 9073 & 38730.85 & 1755.71 & 1.000 & & \\
\hline Ever users & 439 & 9073 & 41638.99 & 1054.30 & 0.596 & $0.529-0.672$ & $<0.0001$ \\
\hline \multicolumn{8}{|c|}{ Tertiles of cumulative duration of metformin therapy (months) } \\
\hline Never users & 680 & 9073 & 38730.85 & 1755.71 & 1.000 & & \\
\hline$<24.9$ & 201 & 2991 & 10317.83 & 1948.08 & 1.086 & $0.928-1.273$ & 0.3037 \\
\hline 24.9-53.9 & 157 & 2995 & 14071.85 & 1115.70 & 0.624 & $0.525-0.743$ & $<0.0001$ \\
\hline$>53.9$ & 81 & 3087 & 17249.30 & 469.58 & 0.265 & $0.210-0.334$ & $<0.0001$ \\
\hline \multicolumn{8}{|l|}{ Women* } \\
\hline Never users & 1079 & 5516 & 22363.36 & 4824.86 & 1.000 & & \\
\hline Ever users & 689 & 5516 & 24903.84 & 2766.64 & 0.568 & $0.517-0.625$ & $<0.0001$ \\
\hline \multicolumn{8}{|c|}{ Tertiles of cumulative duration of metformin therapy (months) } \\
\hline Never users & 1079 & 5516 & 22363.36 & 4824.86 & 1.000 & & \\
\hline$<25.7$ & 326 & 1822 & 6147.16 & 5303.26 & 1.085 & $0.958-1.228$ & 0.2002 \\
\hline $25.7-55.0$ & 226 & 1812 & 8342.91 & 2708.89 & 0.552 & $0.478-0.638$ & $<0.0001$ \\
\hline$>55.0$ & 137 & 1882 & 10413.77 & 1315.57 & 0.270 & $0.226-0.322$ & $<0.0001$ \\
\hline
\end{tabular}


Table 3 Sensitivity analyses estimating hazard ratios for osteoporosis/vertebral fracture for ever vs never users of metformin.

\section{Models}

I. Men aged $<60$ years

II. Men aged $60-69$ years

III. Men aged $70-79$ years

IV. Men aged $\geq 80$ years

$\checkmark$. Women aged $<60$ years*

VI. Women aged $60-69$ years*

VII. Women aged $70-79$ years*

VIII. Women aged $\geq 80$ years*

IX. Excluding two consecutive prescriptions of metformin spanning more than 4 months

$X$. Excluding patients treated with incretin-based therapies during follow-up

$\mathrm{XI}$. Excluding patients treated with insulin at entry and/or during follow-up

XII. Excluding patients treated with sulfonylurea at entry and/or during follow-up

XIII. Excluding patients treated with meglitinide at entry and/or during follow-up

XIV. Excluding patients treated with acarbose at entry and/or during follow-up

$X V$. Excluding patients treated with thiazolidinediones at entry and/or during follow-up

$X V I$. Excluding patients with a diagnosis of cancer during follow-up

XVII. Defining primary outcome as osteoporosis only diagnosed at outpatient clinics or during hospitalization

XVIII. Defining primary outcome as vertebral fracture only diagnosed at outpatient clinics or during hospitalization

XIX. Defining primary outcome as vertebral fracture without a prior diagnosis of osteoporosis diagnosed at outpatient clinics or during hospitalization

$X X$. Defining primary outcome as osteoporosis and/or vertebral fracture as a primary diagnosis during hospitalization

XXI. Defining primary outcome as vertebral fracture with or without a prior diagnosis of osteoporosis during hospitalization

XXII. Defining primary outcome as vertebral fracture without a prior diagnosis of osteoporosis during hospitalization

\section{Haza}

Hazard ratio

0.491

0.587

0.640

0.673

0.469

0.526

0.654

0.703

0.575

0.693

0.584

0.595

0.597

0.618

0.639

0.600

0.614

0.622

0.659

$0.547-0.708$

$0.564-0.770$

0.641

$0.530-0.775$

0.700

0.541-0.907

0.586
$<0.0001$

$<0.0001$

0.0069

P value

$<0.0001$

$<0.0001$

$<0.0001$

0.0338

$<0.0001$

$<0.0001$

$<0.0001$

0.0173

$<0.0001$

$<0.0001$

$<0.0001$

0.0777

$<0.0001$

$<0.0001$

$<0.0001$

$<0.0001$

$<0.0001$

$<0.0001$

0.0018

Hazard ratios were estimated by Cox regression incorporated with the inverse probability of treatment weighting using propensity score. * Models conducted in women included two additional covariates in the estimation of propensity score, that is, 'symptomatic menopausal or female climacteric states' and 'use of estrogen'.

and 1713.20 per 100000 person-years. For ever vs never users, a significant $40 \%$ risk reduction was consistently observed in both sexes together (HR: 0.592, 95\% CI: 0.550-0.638), in men (HR: 0.596, 95\% CI: 0.529-0.672) and in women (HR: 0.568, 95\% CI: 0.517-0.625). In the tertile analysis, patients who used metformin for $<25.5$ months in the first tertile had a slightly increased risk (HR: 1.145, 95\% CI: 1.039-1.260, $P<0.01)$ when both sexes were analyzed together. However, significantly lower risk was observed for patients who had used metformin for a longer duration in the second tertile of 25.5-54.8 months (HR: 0.584, 95\% CI: 0.523-0.652, $P<0.001$ ) and third tertile of $>54.8$ months (HR: 0.263, 95\% CI: 0.228-0.304, $P<0.001)$. The analyses conducted in men and women separately showed similar results but the HRs in the first tertiles were not significant.
The sensitivity analyses shown in Table 3 all consistently supported a lower risk of osteoporosis/ vertebral fracture associated with metformin use. The significant risk reduction could be similarly demonstrated in all four age subgroups of $<60,60-69,70-79$ and $\geq 80$ years in either sex, but the risk reduction attenuated with increasing age (Models I-VIII). The benefit of metformin could be similarly demonstrated after excluding patients who did not refill regularly (Model IX) and the protection of metformin was not affected by the use of other antidiabetic drugs (Models X-XV). Although cancer may affect fracture risk and shorten lifespan, the beneficial effect of metformin remained significant after excluding patients with a diagnosis of cancer (Model XVI). The risk reduction associated with metformin use could be similarly demonstrated for osteoporosis (Model XVII) 
and vertebral fracture (Models XVIII, XIX, XXI and XXII), respectively, and for vertebral fracture without a prior diagnosis of osteoporosis (Model XIX for diagnoses made at outpatient clinics or during hospitalization and Model XXII for diagnoses made during hospitalization). Diagnoses of osteoporosis/vertebral fracture made during hospitalization (Model XX) slightly moved the HR toward unity but remained significant (HR: 0.641, 95\% CI: 0.5300.775, $P<0.0001)$ when compared to the corresponding overall HR when diagnoses were made at the outpatient clinics or during hospitalization (HR: 0.592, 95\% CI 0.550-0.638, $P<0.0001$, Table 2).

Table 4 shows the multivariate-adjusted HRs created by traditional Cox regression for osteoporosis/ vertebral fracture (diagnosed at outpatient clinics or during hospitalization) for users vs non-users of insulin, sulfonylurea, meglitinide, acarbose, rosiglitazone, pioglitazone, immunosuppressants and estrogen (in women only) and for metformin ever vs never users in the respective subgroups of these drugs and their $P$-values for interaction. The use of other antidiabetic drugs or estrogen (women only) had a neutral effect but the use of immuosuppressants was associated with an increased risk (HR: 1.383, 95\% CI: 1.184-1.617, $P<0.0001)$. Metformin was significantly associated with a lower risk in all subgroups of use and non-use of the other drugs except in users of insulin and rosiglitazone. Significant interactions between metformin and insulin, sulfonylurea and pioglitazone could be seen $(P$-values for interaction $<0.05)$. The protective effect of metformin was not observed among users of insulin but could be stronger among users of sulfonylurea or pioglitazone than non-users.

The odds ratios after adjustment for all covariates in Table 1 for prevalent users vs non-users of metformin were 0.629 (95\% CI: 0.579-0.682) for both sexes, 0.639 (95\% CI: 0.563-0.725) for men and 0.598 (95\% CI: 0.537-0.666) for women. The odds ratios after adjustment for selected variables that differed significantly between metformin users and non-users and between patients with and without the outcome were 0.627 (95\% CI: 0.579-0.679) for both sexes (adjusted for hypertension, sulfonylurea,

Table 4 Multivariate-adjusted hazard ratios (created by traditional Cox regression) for osteoporosis/vertebral fracture diagnosed at outpatient clinics or during hospitalization for users vs non-users of some medications other than metformin and for metformin ever vs never users in the respective subgroups of the other medications and their $P$-values for interaction.

\begin{tabular}{|c|c|c|c|c|c|c|c|}
\hline \multirow{2}{*}{$\begin{array}{l}\text { Subgroups of other } \\
\text { medications }\end{array}$} & \multicolumn{3}{|c|}{ Users vs non-users of other medications } & \multicolumn{3}{|c|}{$\begin{array}{l}\text { Metformin ever vs never } \\
\text { users in subgroups }\end{array}$} & \multirow[b]{2}{*}{ P-interaction } \\
\hline & $\mathrm{HR}$ & $95 \% \mathrm{Cl}$ & $P$ value & $\mathrm{HR}$ & $95 \% \mathrm{Cl}$ & $P$ value & \\
\hline \multicolumn{8}{|l|}{ Insulin } \\
\hline No & 1.000 & & & 0.577 & $0.533-0.623$ & $<0.0001$ & $<0.0001$ \\
\hline Yes & 0.980 & $0.824-1.166$ & 0.8209 & 1.124 & $0.746-1.693$ & 0.5758 & \\
\hline \multicolumn{8}{|l|}{ Sulfonylurea } \\
\hline No & 1.000 & & & 0.819 & $0.685-0.980$ & 0.0288 & $<0.0001$ \\
\hline Yes & 1.057 & 0.939-1.190 & 0.3598 & 0.544 & $0.498-0.595$ & $<0.0001$ & \\
\hline \multicolumn{8}{|l|}{ Meglitinide } \\
\hline No & 1.000 & & & 0.598 & $0.553-0.646$ & $<0.0001$ & 0.5148 \\
\hline Yes & 0.942 & $0.796-1.115$ & 0.4894 & 0.615 & $0.462-0.820$ & 0.0009 & \\
\hline \multicolumn{8}{|l|}{ Acarbose } \\
\hline No & 1.000 & & & 0.603 & $0.557-0.653$ & $<0.0001$ & 0.7953 \\
\hline Yes & 0.938 & $0.823-1.070$ & 0.3401 & 0.501 & $0.371-0.677$ & $<0.0001$ & \\
\hline \multicolumn{8}{|l|}{ Rosiglitazone } \\
\hline No & 1.000 & & & 0.602 & $0.558-0.650$ & $<0.0001$ & 0.8968 \\
\hline Yes & 1.128 & $0.918-1.387$ & 0.2514 & 0.668 & $0.394-1.135$ & 0.1358 & \\
\hline \multicolumn{8}{|l|}{ Pioglitazone } \\
\hline No & 1.000 & & & 0.611 & $0.567-0.659$ & $<0.0001$ & 0.0125 \\
\hline Yes & 1.018 & $0.777-1.334$ & 0.8953 & 0.163 & $0.071-0.370$ & $<0.0001$ & \\
\hline \multicolumn{8}{|c|}{ Immunosuppressants use } \\
\hline No & 1.000 & & & 0.605 & $0.560-0.654$ & $<0.0001$ & 0.8481 \\
\hline Yes & 1.383 & $1.184-1.617$ & $<0.0001$ & 0.576 & $0.421-0.789$ & 0.0006 & \\
\hline \multicolumn{8}{|c|}{ Estrogen (women only)* } \\
\hline No & 1.000 & & & 0.613 & $0.545-0.689$ & $<0.0001$ & 0.0748 \\
\hline Yes & 1.093 & $0.956-1.250$ & 0.1923 & 0.489 & $0.411-0.581$ & $<0.0001$ & \\
\hline
\end{tabular}


angiotensin converting enzyme inhibitor/angiotensin receptor blocker, calcium channel blocker and HP infection), 0.641 (95\% CI: 0.566-0.726) for men (adjusted for stroke and dementia) and 0.596 (95\% CI: 0.537-0.662) for women (adjusted for hypertension, sulfonylurea and statin). The corresponding odds ratios estimated after adjustment for all covariates and after adjustment for selected covariates were almost the same for both sexes, for men and for women, respectively, suggesting that the estimates would not be biased by the changes in the covariates used for adjustment.

\section{Discussion}

\section{Main findings}

This study showed a reduced risk of osteoporosis/vertebral fracture associated with metformin use in patients with T2DM in either sex (Table 2). A dose-response pattern could be seen and the beneficial effect was especially significant when metformin had been used for more than approximately 2 years (Table 2 ).

\section{Interactions with other antidiabetic drugs}

We did not observe an independent risk association with any other antidiabetic drugs (Table 4). However, we did observe significant interactions between metformin and insulin, sulfonylurea and pioglitazone, respectively (Table 4).

The beneficial effect of metformin could not be demonstrated in insulin users (Table 4), probably because of hypoglycemia-induced falls associated with insulin use (7). Fracture risk associated with sulfonlyurea is not conclusive $(9,17,30)$. Although the risk did not differ between users and non-users of sulfonylurea (Table 4), we did observe a shift of HR for metformin ever users vs never users away from unity in the subgroup of patients who used sulfonylurea ( $P$-interaction $<0.0001$, Table 4 ). Therefore, when sulfonylurea is used, it is better to include metformin as a combination therapy.

Increased fracture risk associated with thiazolidinediones is mainly observed in postmenopausal women and for fractures of long bones (7). In the present study, both rosiglitazone and pioglitazone had a neutral effect on osteoporosis/vertebral fracture (Table 4). However, a significant interaction with metformin was observed for pioglitazone but not for rosiglitazone (Table 4). The remarkable shift of HR away from unity among pioglitazone users suggested that the protective effect of metformin would be more prominent when the patients were using pioglitazone.

\section{Differences from earlier studies}

Metformin was associated with a reduced risk of any fracture of $20-30 \%$ in some studies $(8,17,18)$ but not in others $(9,19,20)$. It should be pointed out that the three studies showing a favorable effect studied any fracture and not vertebral fracture and were conducted mainly in Caucasians $(8,17,18)$. Two of the three studies that did not show a favorable effect used either a cross-sectional design in the Japanese study (494 men and 344 postmenopausal women with T2DM) (9) or a nested case-control design in the Italian study (83 cases and 249 matched controls) (19). Prevalent user bias could not be excluded and both had small sample sizes. The third study was conducted in the USA (20). Although a prospective follow-up design was used, it enrolled only males and evaluated non-vertebral fracture in a small sample size of 779 participants with diabetes mellitus (20). Therefore, a non-significant result in these studies could probably be due to the inadequate study designs or the lack of sufficient statistical power.

\section{Findings supporting early intervention in diabetes}

Patients with T2DM have an increased risk of vertebral fracture and its occurrence indicates a significantly higher risk of non-vertebral fracture and mortality (31). The International Osteoporosis Foundation recommended to take action in the diabetes patients when a T-score is $<-2.0$ or when the patients develop hip or vertebral fracture (32). As shown in Fig. 1, the high proportion (approximately one-third) of an outcome of vertebral fracture, which is predictive for future fracture and mortality (31), indicated a need for early diagnosis and intervention of osteoporosis/ vertebral fracture. Furthermore, only a small proportion of the incident cases would have a prior diagnosis of osteoporosis as a warning sign before the occurrence of vertebral fracture $(5.1 \%$ in ever users and $6.6 \%$ in never users, Fig. 1) and a high proportion would suffer from vertebral fracture without a warning sign of osteoporosis (15.6\% in ever users and $16.0 \%$ in never users, Fig. 1). These findings strongly implied either a late diagnosis of osteoporosis in current medical care or the requirement of a loosening of the diagnostic criteria for osteoporosis in patients with T2DM, for example, a T-score $<-2.0$ as recently recommended by the International Osteoporosis Foundation (32). 


\section{Perspectives}

This observational study has some clinical implications. First, because metformin is safe and not expensive, it may be a good preventive agent for osteoporosis/vertebral fracture and may be used as an adjuvant therapy, in either the diabetes patients or non-diabetes people. Second, whether there can be synergistic effects of metformin and other antiosteoporosis medications are worthy of intensive investigation. Third, the more remarkable effect observed in the younger age groups (Models I-VIII, Table 3) suggested that prescription of metformin to younger patients would result in a more significant impact with better cost-effectiveness.

\section{Potential mechanisms}

Some biological actions of metformin could explain the reduced risk of osteoporosis/vertebral fracture associated with metformin use. First, metformin can reverse the deleterious effects of hyperglycemia on bone metabolism by lowering blood glucose levels (16). Second, metformin itself may exert osteogenic effects (33) and inhibit osteoclasts (15) through the activation of 5'-AMP-activated protein kinase. Third, type 1 collagen synthesis and osteocalcin expression are both stimulated by metformin $(15,16)$ and metformin may stimulate osteoprotegerin, which in turn inhibit the activity of osteoclasts and prevent bone loss (34). Fourth, insulin resistance and inflammation may disturb bone metabolism (16) and metformin may improve insulin resistance and reduce tissue inflammation (35). Fifth, osteoporosis and vertebral fracture are both age-related. Recent studies suggested that metformin has an anti-aging effect because it can target senescent cells and prevents the induction of senescenceassociated secretory phenotype (36). An in vitro and in vivo study did show that metformin protected against intervertebral disc degeneration by attenuating apoptosis and senescence in nucleus pulposus cells via autophagy stimulation (37).

\section{Strengths of the study}

The strengths of the study has been extensively discussed in a previous paper that used a similar study design and statistical approach, with special attention to common methodological limitations seen in pharmacoepidemiological studies such as selection bias, prevalent user bias, immortal time bias and confounding by indication (38).

\section{Study limitations}

Study limitations may include a lack of biochemical, humoral and hormonal data, anthropometric factors, lifestyle, physical activity, menstruation history, cigarette smoking, alcohol drinking, dietary pattern, vitamin D and calcium supplements, family history and genetic parameters. Because only ICD-9-CM codes were used and no laboratory data such as dual-energy X-ray absorptiometry, spine X-ray or CT were available, misclassification of osteoporosis and/or vertebral fracture could not be excluded. However, the effects would only have been underestimated if the misclassifications were not differential in ever and never users of metformin (39).

\section{Conclusions}

The findings of the present study support a lower risk of osteoporosis/vertebral fracture associated with metformin use in patients with T2DM, disregarding sexes. Metformin should be especially considered in patients younger than 60 years of age because they can obtain the strongest protection than patients of older ages.

\section{Supplementary materials}

This is linked to the online version of the paper at https://doi.org/10.1530/ EJE-20-0507.

\section{Declaration of interest}

The author declares that there is no conflict of interest that could be perceived as prejudicing the impartiality of this study.

\section{Funding}

The study was supported by the Ministry of Science and Technology (MOST 107-2221-E-002-129-MY3) of Taiwan and the Yee Fong Charity Foundation. The funders had no role in study design, data collection and analysis, decision to publish, or preparation of the manuscript.

\section{Author contribution statement}

Tseng $\mathrm{C} \mathrm{H}$ researched data and wrote manuscript. The guarantor of this paper is Tseng $\mathrm{CH}$.

\section{Acknowledgements}

The study is based in part on data from the National Health Insurance Research Database provided by the Bureau of National Health Insurance, Department of Health and managed by National Health Research Institutes. The interpretation and conclusions contained herein do not represent those of Bureau of National Health Insurance, Department of Health or National Health Research Institutes. 


\section{References}

1 Hamann C, Kirschner S, Günther KP \& Hofbauer LC. Bone, sweet bone - osteoporotic fractures in diabetes mellitus. Nature Reviews: Endocrinology 20128 297-305. (https://doi.org/10.1038/ nrendo.2011.233)

2 Napoli N, Strollo R, Paladini A, Briganti SI, Pozzilli P \& Epstein S. The alliance of mesenchymal stem cells, bone, and diabetes. International Journal of Endocrinology 20142014 690783. (https://doi. org/10.1155/2014/690783)

3 Hough FS, Pierroz DD, Cooper C, Ferrari SL \& IOF CSA Bone and Diabetes Working Group. MECHANISMS IN ENDOCRINOLOGY: Mechanisms and evaluation of bone fragility in type 1 diabetes mellitus. European Journal of Endocrinology 2016174 R127-R138. (https://doi.org/10.1530/EJE-15-0820)

4 Napoli N, Chandran M, Pierroz DD, Abrahamsen B, Schwartz AV, Ferrari SL \& IOF Bone and Diabetes Working Group. Mechanisms of diabetes mellitus-induced bone fragility. Nature Reviews: Endocrinology 201713 208-219. (https://doi.org/10.1038/nrendo.2016.153)

5 Poiana C \& Capatina C. Fracture risk assessment in patients with diabetes mellitus. Journal of Clinical Densitometry 201720 432-443. (https://doi.org/10.1016/j.jocd.2017.06.011)

6 Vestergaard P. Discrepancies in bone mineral density and fracture risk in patients with type 1 and type 2 diabetes - a meta-analysis. Osteoporosis International 200718 427-444. (https://doi.org/10.1007/ s00198-006-0253-4)

7 Cipriani C, Colangelo L, Santori R, Renella M, Mastrantonio M, Minisola S \& Pepe J. The interplay between bone and glucose metabolism. Frontiers in Endocrinology 202011 122. (https://doi. org/10.3389/fendo.2020.00122)

8 Melton 3rd LJ, Leibson CL, Achenbach SJ, Therneau TM \& Khosla S. Fracture risk in type 2 diabetes: update of a population-based study. Journal of Bone and Mineral Research 200823 1334-1342. (https://doi. org/10.1359/jbmr.080323)

9 Kanazawa I, Yamaguchi T, Yamamoto M \& Sugimoto T. Relationship between treatments with insulin and oral hypoglycemic agents versus the presence of vertebral fractures in type 2 diabetes mellitus. Journal of Bone and Mineral Metabolism 201028 554-560. (https://doi. org/10.1007/s00774-010-0160-9)

10 Hidayat K, Du X, Wu MJ \& Shi BM. The use of metformin, insulin, sulphonylureas, and thiazolidinediones and the risk of fracture: systematic review and meta-analysis of observational studies. Obesity Reviews 201920 1494-1503. (https://doi.org/10.1111/obr.12885)

11 Zhu ZN, Jiang YF \& Ding T. Risk of fracture with thiazolidinediones: an updated meta-analysis of randomized clinical trials. Bone 201468 115-123. (https://doi.org/10.1016/j.bone.2014.08.010)

12 Bazelier MT, de Vries F, Vestergaard P, Herings RM, Gallagher AM, Leufkens HG \& van Staa TP. Risk of fracture with thiazolidinediones: an individual patient data meta-analysis. Frontiers in Endocrinology 20134 11. (https://doi.org/10.3389/fendo.2013.00011)

13 Zhou Z, Jardine M, Perkovic V, Matthews DR, Mahaffey KW, de Zeeuw D, Fulcher G, Desai M, Oh R, Simpson R et al. Canagliflozin and fracture risk in individuals with type 2 diabetes: results from the CANVAS Program. Diabetologia 201962 1854-1867. (https://doi. org/10.1007/s00125-019-4955-5)

14 Hruska KA, Sugatani T, Agapova O \& Fang Y. The chronic kidney disease - mineral bone disorder (CKD-MBD): advances in pathophysiology. Bone 2017100 80-86. (https://doi.org/10.1016/j. bone.2017.01.023)

15 Bahrambeigi S, Yousefi B, Rahimi M \& Shafiei-Irannejad V. Metformin; an old antidiabetic drug with new potentials in bone disorders. Biomedicine and Pharmacotherapy 2019109 1593-1601. (https://doi.org/10.1016/j.biopha.2018.11.032)

16 Jiating L, Buyun J \& Yinchang Z. Role of metformin on osteoblast differentiation in type 2 diabetes. BioMed Research International 2019 2019 9203934. (https://doi.org/10.1155/2019/9203934)
17 Vestergaard P, Rejnmark L \& Mosekilde L. Relative fracture risk in patients with diabetes mellitus, and the impact of insulin and oral antidiabetic medication on relative fracture risk. Diabetologia 200548 1292-1299. (https://doi.org/10.1007/s00125-005-1786-3)

18 Starup-Linde J, Gregersen S \& Vestergaard P. Associations with fracture in patients with diabetes: a nested case-control study. BMJ Open 20166 e009686. (https://doi.org/10.1136/bmjopen-2015-009686)

19 Monami M, Cresci B, Colombini A, Pala L, Balzi D, Gori F, Chiasserini V, Marchionni N, Rotella CM \& Mannucci E. Bone fractures and hypoglycemic treatment in type 2 diabetic patients: a case-control study. Diabetes Care 200831 199-203. (https://doi. org/10.2337/dc07-1736)

20 Napoli N, Strotmeyer ES, Ensrud KE, Sellmeyer DE, Bauer DC, Hoffman AR, Dam TT, Barrett-Connor E, Palermo L, Orwoll ES et al. Fracture risk in diabetic elderly men: the MrOS study. Diabetologia 201457 2057-2065. (https://doi.org/10.1007/s00125-014-3289-6)

21 Salari-Moghaddam A, Sadeghi O, Keshteli AH, Larijani B \& Esmaillzadeh A. Metformin use and risk of fracture: a systematic review and meta-analysis of observational studies. Osteoporosis International 201930 1167-1173. (https://doi.org/10.1007/s00198019-04948-1)

22 Tseng CH. Metformin is associated with a lower risk of colorectal cancer in Taiwanese patients with type 2 diabetes: a retrospective cohort analysis. Diabetes and Metabolism 201743 438-445. (https:// doi.org/10.1016/j.diabet.2017.03.004)

23 Parsons LS. Performing a 1:N case-control match on propensity score. (available at: http://www.google.com.tw/url?sa=t\&rct=j\&q=\&esrc $=s \&$ source $=$ web\&cd $=1 \&$ ved $=0 \mathrm{CBsQFjAAahUKEwibi7HllcnIAhUDoJQKHV}$ eZA9A\&url=http\%3A\%2F\%2Fwww2.sas.com\%2Fproceedings\%2Fsug i29\%2F165-29.pdf\&usg=AFQjCNFOHGWYu8E8Bn4-Bo1TUiJKt T987Q). Accessed on 5 March 2020.

24 Chang L. A study of validation on comorbidity derived from claims data. Master thesis, National Yang-Ming University, 2004. (available at: http://etd.lib.nctu.edu.tw/cgi-bin/gs32/ymgsweb.cgi/ccd=ji3XTg/ search\#result). Accessed on 22 February 2020.

25 Tseng CH. Diabetes, metformin use, and colon cancer: a populationbased cohort study in Taiwan. European Journal of Endocrinology 2012 167 409-416. (https://doi.org/10.1530/EJE-12-0369)

26 Tseng CH. Diabetes, insulin use and Helicobacter pylori eradication: a retrospective cohort study. BMC Gastroenterology 20121246. (https://doi.org/10.1186/1471-230X-12-46)

27 Austin PC \& Stuart EA. Moving towards best practice when using inverse probability of treatment weighting (IPTW) using the propensity score to estimate causal treatment effects in observational studies. Statistics in Medicine 201534 3661-3679. (https://doi. org/10.1002/sim.6607)

28 Austin PC. The performance of different propensity score methods for estimating marginal hazard ratios. Statistics in Medicine 201332 2837-2849. (https://doi.org/10.1002/sim.5705)

29 Schisterman EF, Cole SR \& Platt RW. Overadjustment bias and unnecessary adjustment in epidemiologic studies. Epidemiology 2009 20 488-495. (https://doi.org/10.1097/EDE.0b013e3181a819a1)

30 Zhang Z, Cao Y, Tao Y, E M, Tang J, Liu Y \& Li F. Sulfonylurea and fracture risk in patients with type 2 diabetes mellitus: a metaanalysis. Diabetes Research and Clinical Practice 2020159107990. (https://doi.org/10.1016/j.diabres.2019.107990)

31 Koromani F, Oei L, Shevroja E, Trajanoska K, Schoufour J, Muka T, Franco OH, Ikram MA, Zillikens MC, Uitterlinden AG et al. Vertebral fractures in individuals with type 2 diabetes: more than skeletal complications alone. Diabetes Care 202043 137-144. (https://doi. org/10.2337/dc19-0925)

32 Ferrari SL, Abrahamsen B, Napoli N, Akesson K, Chandran M, Eastell R, El-Hajj Fuleihan G, Josse R, Kendler DL, Kraenzlin M et al. Diagnosis and management of bone fragility in diabetes: an emerging challenge. Osteoporosis International 201829 2585-2596. (https://doi.org/10.1007/s00198-018-4650-2) 
33 Molinuevo MS, Schurman L, McCarthy AD, Cortizo AM, Tolosa MJ, Gangoiti MV, Arnol V \& Sedlinsky C. Effect of metformin on bone marrow progenitor cell differentiation: in vivo and in vitro studies. Journal of Bone and Mineral Research 201025 211-221. (https://doi. org/10.1359/jbmr.090732)

34 Mai QG, Zhang ZM, Xu S, Lu M, Zhou RP, Zhao L, Jia CH, Wen ZH, Jin DD \& Bai XC. Metformin stimulates osteoprotegerin and reduces RANKL expression in osteoblasts and ovariectomized rats. Journal of Cellular Biochemistry 2011112 2902-2909. (https://doi.org/10.1002/ jcb.23206)

35 Hur KY \& Lee MS. New mechanisms of metformin action: focusing on mitochondria and the gut. Journal of Diabetes Investigation 20156 600-609. (https://doi.org/10.1111/jdi.12328)

36 Khosla S, Farr JN, Tchkonia T \& Kirkland JL. The role of cellular senescence in ageing and endocrine disease. Nature Reviews:
Endocrinology 202016 263-275. (https://doi.org/10.1038/s41574-0200335-y)

37 Chen D, Xia D, Pan Z, Xu D, Zhou Y, Wu Y, Cai N, Tang Q, Wang C, Yan $\mathrm{M}$ et al. Metformin protects against apoptosis and senescence in nucleus pulposus cells and ameliorates disc degeneration in vivo. Cell Death and Disease 20167 e2441. (https://doi.org/10.1038/ cddis.2016.334)

38 Tseng $\mathrm{CH}$. Metformin reduces risk of benign nodular goiter in patients with type 2 diabetes mellitus. European Journal of Endocrinology 2019180 365-372. (https://doi.org/10.1530/ EJE-19-0133)

39 Kesmodel US. Information bias in epidemiological studies with a special focus on obstetrics and gynecology. Acta Obstetricia and Gynecologica Scandinavica 201897 417-423. (https://doi.org/10.1111/ aogs.13330)

Received 12 May 2020

Revised version received 27 October 2020

Accepted 30 November 2020 